\section{Mesoporous Silica Nanoparticles Facilitate Antireflective Coating Applications}

Mesoporous silica nanoparticles (MSNs) embedded in silica or other polymer matrices to produce nanocomposite films are promising for their use in applications requiring low optical index of refraction. However, their use has been limited for these applications because the removal of the surfactants from the mesostructured silica-surfactant composite nanoparticles is required without loss of monodispersity and the internal mesostructure. Modification of the inter-
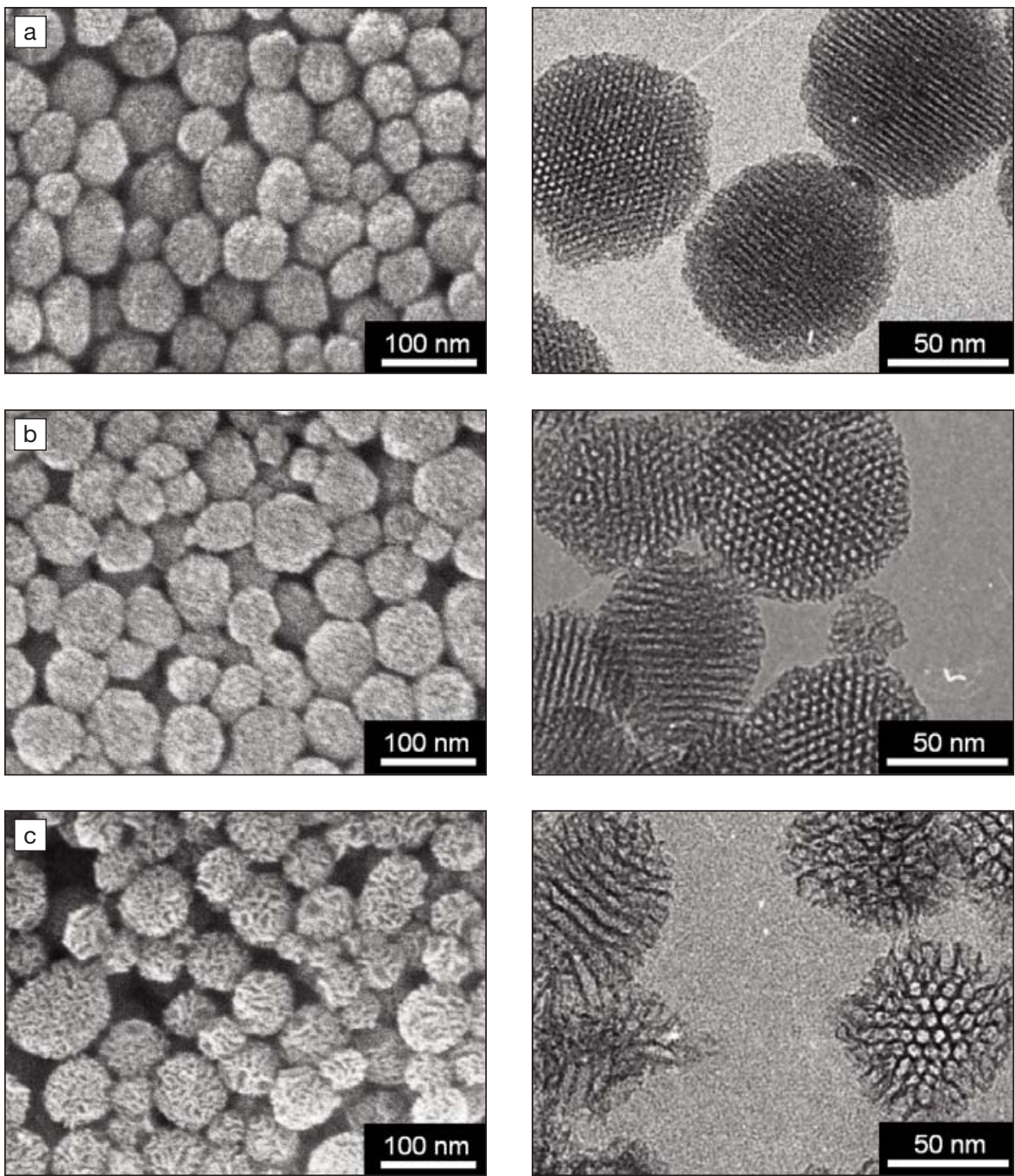

Figure 1. The field-emission scanning electron microscopic images (left) and transmission electron microscopic images (right) of trimethylsilylated-mesoporous silica nanoparticles (TMS-MSNs) prepared with 1,3,5-trimethylbenzene/cetyltrimethylammonium bromide ratios of (a) 0, (b) 2, and (c) 4. Reproduced with permission from Chem. Mater. 22 (1) (2010) 12; DOl: 10.1021/cm902239a @ 2010 American Chemical Society. ite films formed by such nanoparticles embedded in a silica matrix.

As reported in the January 12 issue of Chemistry of Materials (DOI: 10.1021/ cm902239a; p. 12), mesoporous silica-surfactant composite nanoparticles were prepared by stirring at $333 \mathrm{~K}$ for $4 \mathrm{~h}$ a mixture of tetraethoxysilane (TEOS), 3-aminopropyltriethoxysilane (APTES), cetyltrimethylammonium bromide (CTAB), water, ammonia, and ethylene glycol (EG). To enhance the porosity of the nanoparticles, 1,3,5-trimethylbenzene (TMB) was added as a swelling agent at the beginning of the reaction. To achieve direct trimethlylisation of the nanoparticles the researchers have developed a new process based on the modified Lentz method where hexamethyldisiloxane (HMDS) is used as the silylation agent under highly acidic conditions. The researchers found that after their procedure, trimethylsilylation of the silanol groups on both the internal and external surfaces of the silica-surfactant composite nanoparticles is achieved, leading to the formation of trimethylsilylated MSNs (TMS-MSNs) (see Figure 1). Scanning electron microscopy images of TMSMSNs show that particle sizes are in the range of $40-80 \mathrm{~nm}$ and are almost unchanged by the addition of TMB; however, there is a significant increase in pore diameter when the TMB concentration is increased. Dynamic light scattering measurements show that the TMS-MSNs have excellent dispersibility in alcohols. Therefore, the research team concluded that the trimethylsilylation process is effective, not only for the removal of surfactant while retaining highly ordered mesostructures but also for the long-term stability of the dispersion in alcohol.

Furthermore, nanocomposite films formed by the nanoparticles embedded in a silica matrix were prepared on a glass substrate. The films were 100-120 nm thick and showed a reflectivity lower than that of a silica film, which decreases with the increase in pore size and content of the TMS-MSNs in the film. The researchers said that the minimal reflectivities in the visible-near-infrared wavelength range found for the best films on a glass substrate $(1.5-2.5 \%)$ tentatively meet the requirements for antireflecting coatings. The researchers are now focusing their efforts to further enhance the performance of the composite film based on precise tuning of the particle size, porosity, and the loading amount of MSNs in the matrix.

Rosalía SERNA 